\title{
Estudos bibliométricos sobre a produção científica da temática Tecnologias de Informação e Comunicação em bibliotecas
}

\author{
Bibliometric studies about the scientific production of the thematic information and \\ communication technologies in libraries
}

Charles Rodrigues Doutorando do Programa de Pós-Graduação em Ciência da Informação da Universidade Federal de Santa Catarina - UFSC.

Bibliotecário da Biblioteca Pública de Itajaí - SC. E-mail: falecomcharles@yahoo.com.br

\begin{abstract}
Angel Freddy Godoy Viera
Doutor em Engenharia de Produção pela Universidade Federal de Santa Catarina - UFSC. Professor do Programa de Pós-Graduação em Ciência da Informação da Universidade Federal de Santa Catarina.

E-mail: godoy@cin.ufsc.br
\end{abstract}

\begin{abstract}
Resumo
Para medir a atividade científica, tem-se utilizado os indicadores bibliográficos que se baseiam em análise estatística de dados quantitativos encontrados na produção técnica e científica. O objetivo deste estudo foi delinear um panorama da produção científica da temática Tecnologias de Informação e Comunicação em bibliotecas, de modo a identificar: os autores mais produtivos (Lei de Lotka); a evolução histórica do número de publicações e os periódicos que mais publicaram sobre a temática (Lei de Bradford); e as principais abordagens que cobrem o tema (Lei de Zipf). A metodologia seguiu quatro etapas: escolha da Web of Science como base de dados de consulta; configuração dos parâmetros das estratégias de busca e do período de cobertura; depuração dos resultados; e tratamento dos dados de pesquisa. Os resultados mostraram que os primeiros trabalhos indexados na Web of Science datam de 1988 e que, até 2014, foram produzidos 458 artigos. A produção científica se mostrou estável nos últimos anos. Os autores mais produtivos foram Gomez, Fourie e Aharony. Não se observou uma alta concentração em um grupo específico, mas sim uma ampla difusão de autores. Identificou-se que são cinco os periódicos com maior produtividade, responsáveis por um terço do total da produção científica: Electronic Library, Program Electronic Library and Information Systems, Library Hi Tech, Library Trends e Libri. As principais palavras-chave indexadas pela Web of Science foram: Academic Library, Internet, Digital Library, Information Retrieval, Librarians e Mobile Services. São abordagens muito presentes no desenvolvimento de produtos, serviços, softwares e aplicativos na sociedade atual.
\end{abstract}

Palavras-chave: Estudos bibliométricos. Lei de Lotka. Lei de Bradford. Lei de Zipf. Tecnologia de Informação e Comunicação. Bibliotecas.

\begin{abstract}
To measure the scientific activity, it has used the bibliographic indicators that are based on statistical analysis of quantitative data found in scientific and technical production. The aim of this study was to delineate an overview of the scientific production of the theme Information and Communication Technologies in libraries, in order to identify: the most productive authors (Lotka's Law); the historical development of number of publications and journals that published on the subject (Bradford Law); and the main approaches that cover the topic (Zipf's Law). The methodology followed four steps: choice of Web of Science as the basis for query data; setting the parameters of search strategies and the coverage period; clearance of the results; and processing of survey data. The results showed that the first works indexed in the Web of Science dating back to 1988 and that by 2014, 458 articles were produced. The scientific production remained stable in recent years. The most productive authors were Gomez, Fourie and Aharony. There was no a high concentration in a particular group, but rather a widespread authors. It was identified that there are five journals with higher productivity, account for one third of the total scientific output: Electronic Library, Program Electronic Library and Information Systems, Library Hi Tech, Library Trends and Libri. The main keywords indexed by Web of Science were: Academic Library, Internet, Digital Library, Information Retrieval, Librarians and Mobile Services. They are very present approaches in product development, services, software and applications in today's society.
\end{abstract}

Keywords: Bibliometric studies. Lotka's Law. Bradford`s Law. Zipf`s Law. Information and Communication Technology. Libraries.

InCID: R. Ci. Inf. e Doc., Ribeirão Preto, v. 7, n. 1, p. 167-180, mar./ago. 2016.

DOI: 10.11606/issn.2178-2075.v7i1p167-180 
Estudos bibliométricos sobre a produção científica da temática Tecnologias de Informação e Comunicação em bibliotecas

\section{Introdução}

O estudo da produção científica sobre a temática Tecnologias de Informação $e$ Comunicação (TICs) em bibliotecas é relevante, pois, ao se observar o contexto tecnológico atual, pode-se verificar: a tendência às convergências tecnológicas de equipamentos e conteúdos; o número expressivo de dispositivos e conexões móveis no mundo, em torno de mais de 7 bilhões em maio de 2015 (GSMA Intelligence, 2015); a conversão por meio de digitalização de livros impressos e outros conteúdos que já nasceram em formato digital; e, paralelamente, o aumento do número de usuários considerados nativos digitais ${ }^{1}$.

É necessário que os serviços oferecidos pelas bibliotecas estejam adequados a esse cenário tecnológico, atendendo a uma demanda de usuários altamente proficientes com o ambiente digital, hábeis em multitarefas e aptos a aceitar uma quantidade enorme de informações. Esses usuários também estão mais familiarizados com computadores do que com livros em papel, já que buscam respostas rápidas e interações imediatas por meio das redes de computadores.

Nesse ambiente (biblioteca), em que o processo de transformação dos objetos (acervo) e dos objetivos (atendimento à demanda informacional dos usuários) ocorre contextualizado num estado de imperativo tecnológico (Tecnologias de Informação e Comunicação), estudos bibliométricos podem fornecer subsídios (menos enviesados) para o direcionamento de esforços em pesquisas e tomadas de decisão.

A necessidade de avaliar a produção científica e seu impacto na sociedade visa fundamentalmente a adequar, de modo conveniente, os recursos destinados à pesquisa e ao desenvolvimento a fim de que funcionem, assim, como instrumentos para o planejamento de políticas públicas na área da ciência, gerenciamento de pesquisas e tomada de decisões neste setor (SANCHO, 1990; MACIAS-CHAPULA, 1998; SANTOS, 2003).

Para medir a atividade científica, tem-se utilizado os indicadores bibliográficos que se baseiam em análise estatística de dados quantitativos encontrados na produção técnica e científica. Por meio desses indicadores, pode-se, entre outros aspectos, determinar: (a) o crescimento quantitativo e qualitativo da literatura; (b) o envelhecimento dos campos

\footnotetext{
${ }^{1} \mathrm{O}$ termo nativos digitais se refere à geração que nasceu depois de 1980 e passou a maior parte de sua adolescência durante a revolução digital. Essa geração se formou na faculdade, trocou pelo menos 200.000 mensagens de texto e e-mails, gastou pelo menos 10.000 horas jogando videogames e pelo menos 10.000 horas em telefones celulares (PRENSKY, 2001).
}

InCID: R. Ci. Inf. e Doc., Ribeirão Preto, v. 7, n. 1, p. 167-180, mar./ago. 2016. 
científicos e a obsolescência da informação e dos paradigmas científicos; (c) a dinâmica e estrutura da comunicação científica (principalmente a formal); (d) a produtividade dos autores e instituições medida pela quantidade de trabalhos publicados; (e) a colaboração entre cientistas e/ou instituições medida pelo número de autores por artigo ou centros de pesquisa colaboradores (sobretudo baseados em coautoria); (f) os estudos de citação, fator de impacto; (g) a análise e avaliação de fontes difusoras dos trabalhos por meio de indicadores de impacto das fontes; (h) a dispersão de publicações científicas entre várias fontes; (i) as características e funções de diversos tipos documentais (literatura branca e cinzenta); (j) as relações interdisciplinares, intradisciplinares e multidisciplinares na ciência; (l) a evolução de disciplinas, subdisciplinas e novos conceitos; e (m) as características de frequência de ocorrência de palavras em textos (SANCHO, 1990; NORONHA; MARICATO, 2008).

Ao longo do século $\mathrm{XX}$, estudos métricos foram desenvolvidos e aperfeiçoados. A literatura especializada (QUONIAM et al., 2001; URBIZAGÁSTEGUI ALVARADO, 2002; BUFREM e PRATES, 2005; GUEDES; BORSCHIVER, 2005; ARAÚJO, 2006; NORONHA; MARICATO, 2008; PINTO; GONZALES-AGUILAR, 2014) aponta três principais leis bibliométricas: a primeira, a Lei de Lotka de 1926 (relação do quadrado inverso de autoria), propõe medir a produtividade dos autores; a segunda, a Lei de Bradford de 1934 (dispersão da produtividade), visa medir a dispersão do conhecimento científico em publicações periódicas; e a terceira, Lei de Zipf de 1949 (menor esforço em terminologias), objetiva aferir a distribuição de frequências de palavras de um texto.

A Lei de Lotka analisa a produtividade científica dos autores, ou seja, verifica a contribuição de cada um para o desenvolvimento científico em sua área de conhecimento. Lotka (1926) formulou os princípios da lei do quadrado inverso, expondo que a quantidade de autores que produzem $n$ contribuições em um determinado campo científico é aproximadamente 1/n2 daqueles que geram apenas uma contribuição e que a proporção daqueles que geram uma única contribuição é em torno de $60 \%$. Tal lei foi aperfeiçoada por Price, por meio de estudos realizados entre 1965 e 1971, o qual concluiu que 1/3 da literatura é produzida por menos de $1 / 10$ dos autores mais produtivos (média de 3,5 documentos/autor) e que $60 \%$ dos autores produzem um único documento (QUONIAM et al., 2001; URBIZAGÁSTEGUI ALVARADO, 2002; GUEDES; BORSCHIVER, 2005; ARAÚJO, 2006; NORONHA; MARICATO, 2008; PINTO; GONZALES-AGUILAR, 2014).

A Lei de Bradford permite calcular o grau de relevância dos periódicos em uma 
determinada área do conhecimento. Bradford, adotando o periódico para a sua análise devido às características de incidência de assuntos e tendências, observou que poucos periódicos produzem muitos artigos (supostamente de maior qualidade ou relevância) e muitos periódicos produzem poucos artigos. O enunciado da Lei de Bradford mostra que se uma grande coleção de periódicos for disposta em ordem decrescente de produtividade de artigos de um determinado assunto, pode-se identificar um cluster central de títulos que tratam essencialmente dessa temática e outros clusters que incluem o mesmo número de artigos do cluster central detentor do maior número de títulos. Assim, para a determinação dos clusters dos títulos produtivos, o total de artigos deve ser somado e divido por três; dessa forma, em uma lista decrescente de produtividade dos títulos, aparecem três clusters, cada um contendo $1 / 3$ do total de artigos relevantes. O primeiro cluster (central) contém um reduzido número de periódicos altamente produtivos; o segundo contém um número maior de periódicos menos produtivos; e o terceiro inclui um número maior de periódicos com menor produtividade (QUONIAM et al., 2001; GUEDES; BORSCHIVER, 2005; ARAÚJO, 2006; NORONHA; MARICATO, 2008; PINTO; GONZALES-AGUILAR, 2014).

A Lei de Zipf permite estimar as frequências de ocorrência das palavras de um determinado texto científico e tecnológico. Zipf formulou o princípio do menor esforço: existe uma economia do uso de palavras, o que significa que, se a tendência é usar o mínimo, elas não vão se dispersar, pelo contrário, uma mesma palavra vai ser usada muitas vezes, e as palavras mais usadas indicam o assunto do documento. Sua proposta, assim, é a de que, se forem listadas as palavras que ocorrem num texto em ordem decrescente de frequência, a posição de uma palavra na lista multiplicada por sua frequência é igual a uma constante. A equação para esse relacionamento é: $\mathrm{r}$ x f = k, em que r é a posição da palavra, f é a sua frequência e k é a constante (QUONIAM et al., 2001; GUEDES; BORSCHIVER, 2005; ARAÚJO, 2006; NORONHA; MARICATO, 2008; PINTO; GONZALES-AGUILAR, 2014).

Quoniam et al (2001), citando um trabalho de 1992, explicam a curva de Zipf, que é dividida em três zonas de distribuição: a Zona I - Informação trivial ou básica define os temas centrais da análise bibliométrica; a Zona II - Informação interessante se localiza entre as Zonas $I$ e $I I I$ e mostra ora os temas periféricos, ora a informação potencialmente inovadora - é aí que as transferências de tecnologia relacionadas aos novos temas devem ser consideradas; e a Zona III - Ruído tem como característica possuir conceitos ainda não emergentes, sendo impossível afirmar se eles serão emergentes ou se são apenas ruídos estatísticos. 
Conforme exposto, os estudos métricos apontam caminhos às novas pesquisas, pois identificam lacunas na literatura especializada e atualizam encaminhamentos teóricos e metodológicos mais precisos. Dessa forma, o objetivo deste estudo é delinear um panorama da produção científica da temática Tecnologias de Informação e Comunicação em bibliotecas, de modo a identificar: (a) os autores mais produtivos (Lei de Lotka - relação do quadrado inverso de autoria); (b) a evolução histórica do número de publicações e os periódicos que mais publicaram sobre a temática (Lei de Bradford - dispersão da produtividade); e (c) as principais abordagens que cobrem o tema (Lei de Zipf-menor esforço em terminologias).

\section{Metodologia}

Como meio a alcançar os objetivos propostos, a metodologia seguiu quatro etapas sequenciais.

Para a primeira etapa, foi escolhida a Web of Science como base de dados de consulta. Esta foi adotada por sua cobertura global e completa dos assuntos propostos, reconhecidamente estruturada para análise de informação para a produção de indicadores, sem necessidade de grandes manipulações prévias dos dados (SANTOS, 2003). A Web of Science é uma base de dados do Instituto de Informação Científica (ISI) que indexa a Science Citation Index Expanded (SCI-EXPANDED), desde 1945 até o presente, a Social Sciences Citation Index (SSCI), de 1956 até o presente, e a Arts \& Humanities Citation Index (A\&HCI), desde 1975 até o presente (PINTO; GONZALES-AGUILAR, 2014).

Na segunda etapa, foi definida a configuração dos parâmetros das estratégias de busca e o período de cobertura. Para tanto, foram empregados os descritores information and communication and technolog* and librar*, utilizando-se o campo de pesquisa Tópico, que engloba a consulta ao título, o resumo e as palavras-chave. Os resultados foram limitados às categorias da Web of Science: Information Science Library Science, Computer Science Information Systems e Education Educational Research, refinando os resultados apenas ao tipo de documento artigo. O período de cobertura da pesquisa abrangeu de 1945 até 2014. Dessa forma, o universo da pesquisa se concentrou em 458 artigos de periódicos recuperados na Web of Science.

Na terceira etapa, ocorreu a depuração dos resultados. Para isso, foram retirados os possíveis documentos duplicados, os sem aderência à pesquisa e os fora do escopo da temática. 
Estudos bibliométricos sobre a produção científica da temática Tecnologias de Informação e Comunicação em bibliotecas

$\mathrm{Na}$ quarta etapa, executou-se o tratamento dos dados de pesquisa, mediante exportação e tabulação dos resultados. Utilizaram-se técnicas e ferramentas, como Excel da Microsoft Office e Bloco de Notas, permitindo, assim, visualizar os resultados e criar tabelas e imagens.

\section{Resultados e Discussões}

Como o objetivo principal deste estudo foi delinear um panorama da produção científica da temática Tecnologias de Informação e Comunicação em Bibliotecas, decidiu-se traçar uma linha histórica da produção anual de artigo sobre esse tema, apresentada no Gráfico 1, cujo recorte temporal são os últimos 10 anos.

Gráfico 1 - Produção científica anual sobre a temática Tecnologias de Informação e Comunicação em Bibliotecas

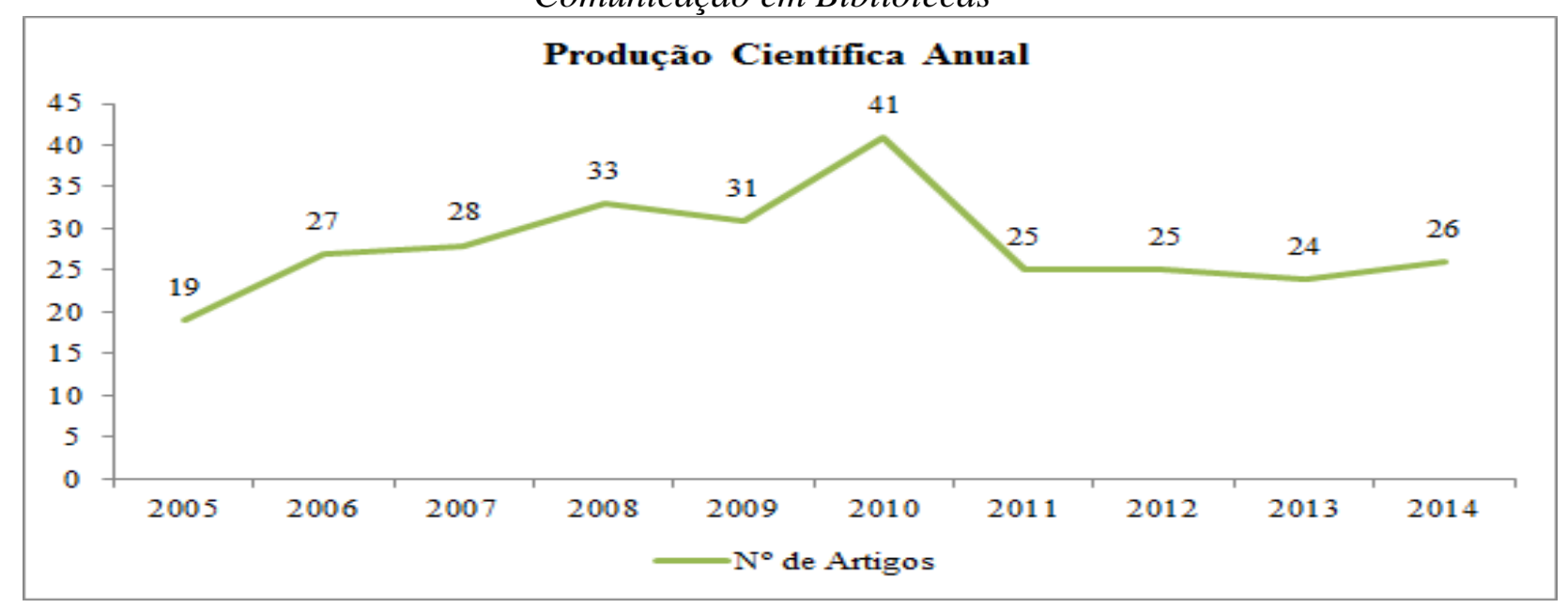

Fonte: Dos autores (2015).

Os primeiros trabalhos sobre a temática deste estudo, indexados na Web of Science, datam de 1988. Em 27 anos, foram publicados 458 artigos, com média de 16,9 artigos/ano. O maior crescimento ocorreu nos últimos 10 anos, já que concentram em torno de $42 \%$ do total da produção. O ano com maior produção foi o de 2010 (41 artigos), embora, no ano seguinte, tenha havido uma queda de aproximadamente 40\% e, nos anos seguintes (2011-2014), tenha se mantido estável, com uma média de 25 artigos por ano.

Na Tabela 1, são apresentados os 13 autores mais produtivos sobre a temática. No total, foram encontrados 761 autores. 
Tabela 1 - Autores mais produtivos

\begin{tabular}{l|c}
\hline Autores & $\mathbf{N}^{\mathbf{0}}$ de Artigos Publicados \\
\hline Gomez, R & 6 \\
\hline Fourie, I & 5 \\
\hline Aharony, N & 4 \\
\hline Mutula, SM & 4 \\
\hline Stilwell, C & 4 \\
\hline Sugimoto, CR & 4 \\
\hline Anwar, MA & 3 \\
\hline Baxter, G & 3 \\
\hline Levy, P & 3 \\
\hline Mahmood, K & 3 \\
\hline Marcella, R & 3 \\
\hline Ramzan, M & 3 \\
\hline Westbrook, L & 3 \\
\hline
\end{tabular}

Fonte: Dos autores (2015).

O autor mais produtivo é Gomez, com seis trabalhos publicados, seguido por Fourie, com cinco, e Aharony, Mutula, Stilwell e Sugimoto, com quatro artigos cada um. Pode-se observar que não há uma alta concentração de publicações em um grupo específico, e sim ampla difusão de autores. Esta é uma característica de uma área do conhecimento cujo referencial teórico, identidade e institucionalização do corpo de autoridade estão em processo de construção. Com os dados apresentados, considera-se inapropriada a aplicação da Lei de Lotka, uma vez que $7,1 \%$ dos autores correspondem a $15,5 \%$ do total das publicações pesquisadas.

$\mathrm{Na}$ Tabela 2, expõem-se os periódicos com maiores números de artigos publicados anualmente, divididos em dois agrupamentos (Clusters). Para a melhor visualização da Tabela, optou-se por apresentar os dados do período de 2005-2014. O primeiro, cluster central, é composto por cinco periódicos responsáveis por um terço das publicações, e o segundo agrupamento, cluster secundário, é constituído por 18 periódicos com produtividade menor do que o primeiro. 
Estudos bibliométricos sobre a produção científica da temática Tecnologias de Informação e Comunicação em bibliotecas

Tabela 2 - Periódicos com maiores números de publicações

\begin{tabular}{|c|c|c|c|c|c|c|c|c|c|c|c|c|c|}
\hline Raking & Periódicos & $\begin{array}{l}1991- \\
2004\end{array}$ & 2005 & 2006 & 2007 & 2008 & 2009 & 2010 & 2011 & 2012 & 2013 & 2014 & Total \\
\hline \multicolumn{14}{|c|}{ Primeiro Agrupamento (Cluster Central) } \\
\hline $1^{\mathrm{o}}$ & $\begin{array}{l}\text { Electronic } \\
\text { Library }\end{array}$ & 15 & 7 & 11 & 7 & 6 & 5 & 7 & 4 & 3 & 2 & 5 & 72 \\
\hline $2^{\circ}$ & $\begin{array}{c}\text { Program } \\
\text { Electronic } \\
\text { Library and } \\
\text { Information } \\
\text { Systems }\end{array}$ & 9 & 2 & 2 & 2 & 4 & 2 & 2 & 1 & 1 & 2 & - & 27 \\
\hline $3^{\circ}$ & $\begin{array}{c}\text { Library Hi } \\
\text { Tech }\end{array}$ & - & - & 1 & 2 & 2 & 5 & 4 & 1 & 3 & 3 & 3 & 24 \\
\hline $4^{\circ}$ & $\begin{array}{l}\text { Library } \\
\text { Trends }\end{array}$ & 9 & - & - & 1 & - & - & 3 & 1 & - & 1 & 2 & 17 \\
\hline $5^{\circ}$ & Libri & 9 & - & 2 & - & 1 & - & - & 3 & - & - & - & 15 \\
\hline \multicolumn{14}{|c|}{ Segundo Agrupamento (Cluster Secundário) } \\
\hline $6^{\circ}$ & $\begin{array}{c}\text { Aslib } \\
\text { Proceedings }\end{array}$ & 6 & - & 2 & 1 & - & 2 & - & - & 1 & 2 & - & 14 \\
\hline $6^{\circ}$ & $\begin{array}{c}\text { Journal Of } \\
\text { Librarianshi } \\
\text { p And } \\
\text { Information } \\
\text { Science }\end{array}$ & 7 & - & 2 & 1 & - & 1 & 1 & - & 1 & 1 & - & 14 \\
\hline $7^{\circ}$ & $\begin{array}{l}\text { Bulletin Of } \\
\text { The Medical } \\
\text { Library } \\
\text { Association }\end{array}$ & 12 & - & - & - & - & - & - & - & - & - & - & 12 \\
\hline $8^{\circ}$ & $\begin{array}{c}\text { Journal Of } \\
\text { Documentati } \\
\text { on }\end{array}$ & 2 & 1 & - & 2 & 2 & 1 & - & 2 & 1 & - & - & 11 \\
\hline $9^{\circ}$ & $\begin{array}{c}\text { Library \& } \\
\text { Information } \\
\text { Science } \\
\text { Research }\end{array}$ & 5 & 1 & - & - & - & 2 & 1 & - & 1 & - & - & 10 \\
\hline $9^{\circ}$ & $\begin{array}{c}\text { Journal Of } \\
\text { Academic } \\
\text { Librarianshi } \\
\text { p }\end{array}$ & 4 & - & - & - & 2 & - & - & - & 1 & 2 & 1 & 10 \\
\hline $10^{\circ}$ & $\begin{array}{c}\text { African } \\
\text { Journal Of } \\
\text { Library } \\
\text { Archives } \\
\text { And } \\
\text { Information } \\
\text { Science }\end{array}$ & - & - & - & 1 & 1 & 2 & 1 & 1 & 1 & 1 & 1 & 09 \\
\hline $10^{\circ}$ & $\begin{array}{c}\text { Health } \\
\text { Information } \\
\text { And } \\
\text { Libraries } \\
\text { Journal } \\
\end{array}$ & - & 1 & - & 1 & 1 & - & 1 & 2 & 2 & - & 1 & 09 \\
\hline $10^{\circ}$ & $\begin{array}{c}\text { Informacao } \\
\& \\
\text { Sociedade- } \\
\text { Estudos }\end{array}$ & - & - & - & - & 4 & 1 & 1 & - & 1 & - & 2 & 09 \\
\hline $10^{\circ}$ & $\begin{array}{l}\text { Information } \\
\text { Research-An } \\
\text { International }\end{array}$ & 1 & - & - & 2 & - & 3 & 1 & - & - & 1 & 1 & 09 \\
\hline
\end{tabular}




\begin{tabular}{c|c|c|c|c|c|c|c|c|c|c|c|c|c}
\hline & $\begin{array}{c}\text { Electronic } \\
\text { Journal }\end{array}$ & & & & & & & & & & & & \\
\hline $10^{\circ}$ & $\begin{array}{c}\text { Journal of } \\
\text { the Medical } \\
\text { Library } \\
\text { Association }\end{array}$ & 2 & 2 & - & 1 & - & - & - & 1 & 1 & 1 & 1 & 09 \\
\hline $11^{\circ}$ & $\begin{array}{c}\text { Information } \\
\text { Developmen } \\
\text { t }\end{array}$ & - & - & - & - & - & 3 & 2 & 1 & 1 & 1 & - & 08 \\
\hline $11^{\circ}$ & $\begin{array}{c}\text { Online } \\
\text { Information } \\
\text { Review }\end{array}$ & 3 & 1 & - & 1 & 1 & - & 1 & - & 1 & - & - & 08 \\
\hline $12^{\circ}$ & $\begin{array}{c}\text { College \& } \\
\text { Research } \\
\text { Libraries }\end{array}$ & 3 & - & 1 & 1 & - & - & - & 1 & - & - & 1 & 07 \\
\hline $12^{\circ}$ & $\begin{array}{c}\text { Information } \\
\text { Technology } \\
\text { And } \\
\text { Libraries }\end{array}$ & 4 & - & - & 1 & - & 1 & 1 & - & - & - & - & 07 \\
\hline $\begin{array}{c}\text { Journal Of } \\
\text { Education } \\
\text { For Library } \\
\text { And } \\
\text { Information } \\
\text { Science }\end{array}$ & 07 & - & - & - & - & - & - & - & - & - & - & 07 \\
\hline $\begin{array}{c}\text { Journal Of } \\
\text { Information } \\
\text { Science }\end{array}$ & 05 & 1 & - & - & 1 & - & - & - & - & - & - & 07 \\
\hline $\begin{array}{c}\text { Journal Of } \\
\text { The } \\
\text { American } \\
\text { Society For } \\
\text { Information } \\
\text { Science And } \\
\text { Technology }\end{array}$ \\
\hline
\end{tabular}

Fonte: Dos autores (2015).

No Cluster Central, os periódicos mais produtivos, por ordem decrescente de número de artigos publicados, são: Electronic Library (72 artigos, representando 15,7\% do total de artigos publicados), Program Electronic Library and Information Systems (27 artigos, representando 5,8\%), Library Hi Tech (24 artigos, representando 5,2\%), Library Trends (17 artigos, representando 3,7\%) e Libri (15 artigos, representando 3,2\%).

Nessa análise, percebe-se a presença da Lei de Bradford, visto que um pequeno número de periódicos (cinco) é responsável por uma expressiva parcela (34\%) do total da produção científica da área, com uma média de 31 artigos por periódico. Um segundo grupo contém um número maior de periódicos (18), representando 36\% da produção, porém com uma média de 9,1 artigos por periódico. E um terceiro grupo, com número maior de periódicos (74), corresponde a $30 \%$ da produção total com média de produtividade baixa de 1,8 artigo por periódico. 
Estudos bibliométricos sobre a produção científica da temática Tecnologias de Informação e Comunicação em bibliotecas

Na Tabela 3, apresenta-se o ranqueamento das dez palavras-chave frequentemente mais indexadas pela Web of Science nos artigos recuperados sobre a temática em estudo. Essas palavras representam a Informação trivial ou básica, definindo, assim, os temas centrais dessa análise bibliométrica.

Tabela 3 - Palavras-chave mais frequentes

\begin{tabular}{l|c}
\hline Palavras-Chave & Frequência \\
\hline Academic Library & 44 \\
\hline Internet & 26 \\
\hline Digital Library & 22 \\
\hline Information Retrieval & 17 \\
\hline Librarians & 17 \\
\hline Mobile Services & 17 \\
\hline Índia & 12 \\
\hline Information Science & 12 \\
\hline Nigéria & 12 \\
\hline Information Literacy & 11 \\
\hline Fonte: Dos autores (2015).
\end{tabular}

No total, foram identificadas 623 palavras-chave utilizadas pela Web of Science para representar os artigos da temática de pesquisa, resultando, ao todo, 1.120 indexações. A palavra que aparece com maior frequência é Academic Library, encontrada 44 vezes. Em seguida, é a palavra Internet, 26 vezes. Após, seguem as palavras-chave Digital Library, 22 vezes, e Information Retrieval, Librarians e Mobile Services, 17 vezes.

Também foram identificadas 462 palavras-chave com apenas uma ocorrência, podendose considerá-las como Ruídos, ou seja, como termos que não possuem um conceito consolidado.

A Figura 2 representa as 37 palavras-chave encontradas com mais frequência. 


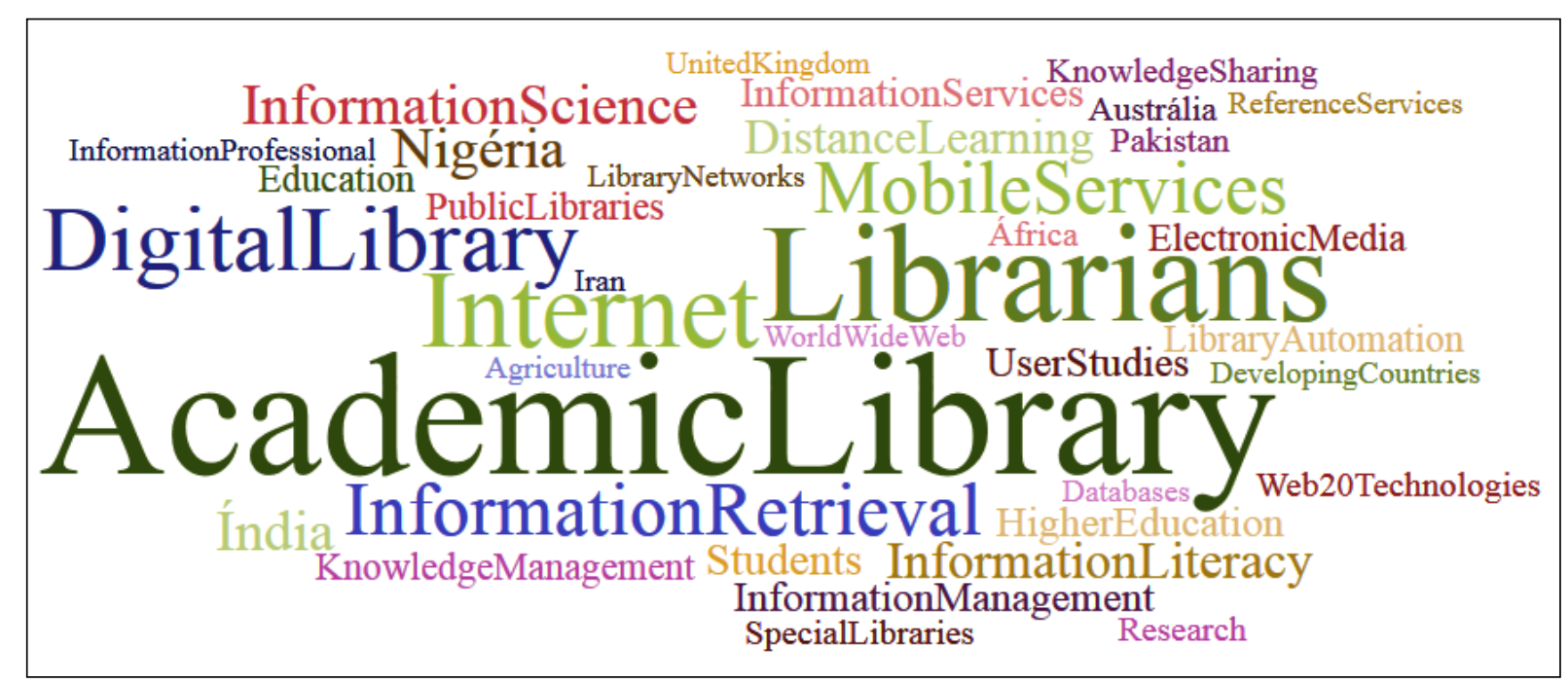

Figura 2 - Nuvem das principais palavras-chave

Fonte: Dos autores (2015).

Diante das palavras-chave que mais representaram os estudos encontrados sobre o tema Tecnologias de Informação e Comunicação em bibliotecas, pode-se inferir que o papel das bibliotecas universitárias é de vanguarda, pois é onde, de certo modo, o desenvolvimento de produtos e inovações é mais profícuo. Outra importante abordagem que predomina é o uso da Internet como meio de fomento e difusão de serviços, produtos e conteúdos das bibliotecas, associada com a preocupação de criação das bibliotecas digitais como forma de oferecer acesso a conteúdos digitais. Dessa forma, percebe-se que os estudos sobre as bibliotecas se alinham e se ajustam aos 'novos' contextos tecnológicos. Outras palavras bastante frequentes Information Retrieval, Librarians e Mobile Services - demonstram que os estudos estão propensos a refletir sobre as demandas atuais dos usuários acerca dos novos desafios e consumos tecnológicos presentes na sociedade. 
Estudos bibliométricos sobre a produção científica da temática Tecnologias de Informação e Comunicação em bibliotecas

\section{Considerações}

Após a análise desenvolvida com os dados dos estudos bibliométricos apresentados, aspectos relevantes foram revelados, a saber.

Estudos sobre a temática Tecnologia de Informação e Comunicação em bibliotecas são cada vez mais necessários, sobretudo em decorrência do nível de desenvolvimento de equipamentos, redes de infraestrutura física e digital, novos modelos de negócios, número de dispositivos móveis, redes sociais virtuais e comportamentos sociais dos usuários diferentes dos estabelecidos até então.

A produção científica sobre a temática desse estudo se mostrou estável nos últimos anos, com uma oscilação maior no ano de 2010. Uma dificuldade encontrada para avaliar esse tema foi a complexidade de variáveis e áreas do conhecimento que se ocupam de estudar esse fenômeno mediante utilização de diversas nomenclaturas para produtos, serviços, softwares e aplicativos, nem sempre indexados adequadamente nas bases de dados.

Os autores mais produtivos encontrados foram Gomez e Fourie, embora não tenham apresentado uma produção muito maior que os demais autores. Dessa forma, não se observou uma alta concentração em um grupo específico, mas sim uma ampla difusão de autores. A característica de ausência de uma identidade e institucionalização de um corpo de autoridade também pode ser explicada pelo grande número de diferentes áreas do conhecimento que tratam da temática.

Já em relação aos periódicos com maior produtividade, encontrou-se uma alta centralização em cinco periódicos principais, responsáveis por um terço do total da produção científica sobre a temática: Electronic Library, Program Electronic Library and Information Systems, Library Hi Tech, Library Trends e Libri.

Sobre as principais palavras-chave indexadas pela Web of Science para representar a temática desse estudo, destacam-se: Academic Library, Internet, Digital Library, Information Retrieval, Librarians e Mobile Services. São abordagens muito presentes nas fases de desenvolvimento de produtos, serviços, softwares e aplicativos na sociedade atual; assim, podese considerar que os estudos estão alinhados a essas "novas" exigências.

No entanto, criticamente, percebe-se carência de estudos que envolvam as seguintes temáticas: modelos de negócio, política de aquisição de conteúdo, plataforma tecnológica, 
gestão dos direitos de propriedade intelectual e preservação digital, temáticas que foram identificadas em outro estudo deste autor (RODRIGUES, 2014).

Por fim, estudos bibliométricos são importantes para consolidar aspectos observados empiricamente e apontar novas possibilidades e rumos da pesquisa científica sobre as Tecnologias de Informação e Comunicação em bibliotecas.

\section{Referências}

ARAÚJO, C. A. Bibliometria: evolução histórica e questões atuais. Em Questão, Porto Alegre, v. 12, n. 1, p. 11-32, jan./jun., 2006. Disponível em:

$<$ http://www.revistas.univerciencia.org/index.php/revistaemquestao/article/viewFile/3707/349 5>. Acesso em: 14 maio 2015.

BUFREM, L.; PRATES, Y. O saber científico registrado e as práticas de mensuração da informação. Ciência da Informação, Brasília, v. 34, n. 2, p. 9-25, 2005. Disponível em: <http://www.scielo.br/pdf/ci/v34n2/28551.pdf >. Acesso em: 14 maio 2015.

GUEDES, V. L. S.; BORSCHIVER, S. Bibliometria: uma ferramenta estatística para a gestão da informação e do conhecimento, em sistemas de informação, de comunicação e de avaliação científica e tecnológica. In: CINFORM - ENCONTRO NACIONAL DE CIÊNCIA DA INFORMAÇÃO, 6., 2005. Anais... Salvador: ICI/UFBA, 2005. Disponível em:

$<$ http://www.feg.unesp.br/ fmarins/seminarios/Material\%20de\%20Leitura/Bibliometria/Artig o\%20Bibliometria\%20-\%20Ferramenta\%20estat\%EDstica\%20VaniaLSGuedes.pdf >. Acesso em: 13 maio. 2015.

GSMA INTELLIGENCE. Definitive data and analysis for the mobile industry. Londres, 2015. Disponível em: <http://gsmaintelligence.com>. Acesso em: 13 maio. 2015.

MACIAS-CHAPULA, C. A. O papel da informetria. Ciência da Informação, Brasília, v. 27, n. 2, 1998. Disponível em: 〈http://revista.ibict.br/index.php/ciinf/article/view/342〉. Acesso em: 14 maio 2015.

NORONHA, D. P.; MARICATO, J. M. Estudos métricos da informação: primeiras aproximaç̃oes. Encontros Bibli, Florianópolis, n. esp., p. 116-128, 2008. Disponível em: $<$ https://periodicos.ufsc.br/index.php/eb/article/view/1518-2924.2008v13nesp1p116/1594〉. Acesso em: 14 maio 2015.

PINTO, A. L.; GONZALES-AGUILAR, A. Visibilidad de los estudios en análisis de redes sociales en América del Sur: su evolución y métricas de 1990-2013. TransInformação, Campinas, v. 26, n.3, p. 253-267, set./dez., 2014. Disponível em: $\langle$ http://www.scielo.br/pdf/tinf/v26n3/0103-3786-tinf-26-03-00253.pdf $>$. Acesso em: 14 maio 2015.

PRENSKY, M. Digital natives, digital immigrants. Part II: do they really think differently? In: ___ On the Horizon. Bingley: MCB University Press, 2001. Disponível em: 
Estudos bibliométricos sobre a produção científica da temática Tecnologias de Informação e Comunicação em bibliotecas

<http://www.marcprensky.com/writing/Prensky\%20-

\%20Digital\%20Natives, \%20Digital\%20Immigrants\%20-\%20Part1.pdf > . Acesso em: 14 maio 2015.

QUONIAM, L. et al. Inteligência obtida pela aplicação de data mining em base de teses francesas sobre o Brasil. Ciência da Informação, Brasília, v. 30, n. 2, p. 20-28, 2001.

RODRIGUES, C. Referenciais teóricos sobre o uso de e-book em bibliotecas públicas brasileiras. Revista Brasileira de Biblioteconomia e Documentação, São Paulo, v. 10, n. 2, p. 100-120, jul./dez., 2014. Disponível em: <http://rbbd.febab.org.br/rbbd/article/view/291/379>. Acesso em: 14 maio 2015.

SANCHO, R. Indicadores bibliométricos utilizados en la evaluación de la ciencia y la tecnología. Revista Española de Documentación Científica, v. 13, n. 3-4, p. 842-865, 1990. Disponível em:

$<$ http://digital.csic.es/bitstream/10261/23694/1/SAD_DIG_IEDCyT_Sancho_Revista\%20Esp a\%C3\%B1ola\%20de\%20Documentacion\%20Cientifica13\%284\%29.pdf >. Acesso em: 14 maio 2015.

SANTOS, R. N. M. Indicadores estratégicos em ciência e tecnologia: refletindo a sua prática como dispositivo de inclusão/exclusão. TransInformação, Campinas, v. 15 (ed. esp.), p. 129140, set./dez. 2003. Disponível em: 〈http://www.scielo.br/pdf/tinf/v15nspe/07.pdf > . Acesso em: 14 maio 2015.

URBIZAGÁSTEGUI ALVARADO, R. A Lei de Lotka na bibliometria brasileira. Ciência da Informação, Brasília, v. 31, n. 2, p. 14-20, 2002. Disponível em:

<http://www.scielo.br/pdf/ci/v31n2/12904.pdf>. Acesso em: 14 maio 2015. 